\title{
Heimann, Thorsten (2017): Klimakulturen und Raum: Umgangsweisen mit Klimawandel an europäischen Küsten
}

\author{
Wiesbaden: Springer VS. 36 Tab., 40 Abb., 422 S.
}

\section{Oskar Marg1 (iD}

Online publiziert: 7. Juni 2017

(c) Springer-Verlag Berlin Heidelberg 2017

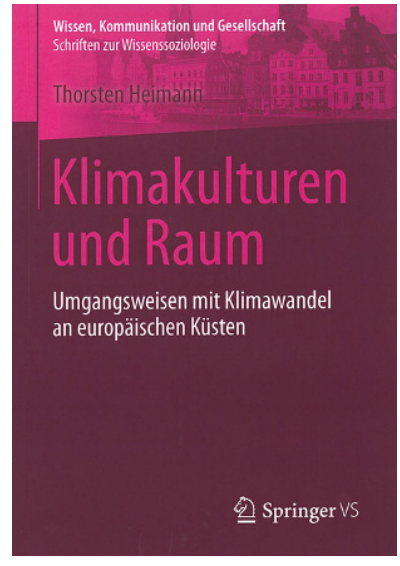

Die unterschiedliche Wahrnehmung der Nutzung von Atomenergie zwischen Deutschland und Frankreich ist ein prominentes Beispiel unter vielen dafür, auf welch unterschiedliche Resonanz ein Umweltthema in verschiedenen Ländern stoßen kann in einem „Rückgriff auf einen gemeinsamen, historisch strukturierten Bestand an kulturellen Deutungen und Symbolisierungen“ (Brand 1995: 51). Bei dieser Perspektive besteht die Gefahr, dass andere Faktoren für unterschiedliche Wahrnehmungen außer Acht gelassen werden. Zudem verändern sich öffentliche Diskurse und die Art der Wahrnehmung im Verlauf der Zeit. So ist es auch bei der Thematik des menschlich verursachten Klimawandels, bei dem der Diskursverlauf „durch wechselseitig sich bedingende Pendelbewegungen von Katastrophismus und Skeptizismus geprägt" gewesen

Dr. Oskar Marg

marg@isoe.de

1 Institut für sozial-ökologische Forschung, Hamburger Allee 45, 60486 Frankfurt am Main, Deutschland ist (Reusswig 2010: 81). Doch welche Faktoren können Unterschiede bei der Wahrnehmung von Klimawandel und Klimaanpassung erklären?

Mit dieser Fragestellung setzt sich Thorsten Heimann in seiner kürzlich erschienenen Dissertation auseinander. In dieser untersucht er zum einen unterschiedliche „Klimakulturen“ - definiert als ,,von Akteuren geteilte Vulnerabilitätsoder Resilienzkonstruktionen“ zum Klimawandel (S. 18) - an europäischen Küsten. Zum anderen geht er der Frage nach, mit welchen Faktoren sich Unterschiede zwischen verschiedenen Klimakulturen erklären lassen. Die Relevanz des Themas begründet sich ihm zufolge dadurch, dass unterschiedliche Wahrnehmungen - ob von Problemen oder Maßnahmen - kollektives Handeln erschweren.

In seinem theoretischen Ansatz (Kapitel 2) geht Heimann von einem sozialkonstruktivistischen Kulturverständnis aus, nach dem Kultur als sozial geteilte Wissensbestände von Akteuren verstanden wird. Hierbei bezieht er sich unter anderem auf Berger und Luckmann (1966), Reckwitz (2000) sowie Knoblauch (2010). Diese geteilten Wissensbestände werden bezogen auf den Aspekt des Klimawandels untersucht, woraus sich der Begriff der Klimakulturen ergibt. Darin werden sowohl die Wahrnehmung von Problemen und Chancen des Klimawandels (Vulnerabilitätskonstruktionen) als auch das Wissen und die Präferenzen über klimabezogene Strategien (Resilienzkonstruktionen) erfasst. Des Weiteren geht Heimann auf den Begriff Raum ein, der in der kulturvergleichenden Forschung oft nur implizit behandelt wird. Als Synthese aus den Begriffen Kultur und Raum entwickelt Heimann den relationalen Kulturraumansatz als eigenes theoretisches Konzept, mit dem er Antworten auf seine Fragen finden will. Kulturen werden demnach verstanden als ,,relationale Anordnungen von Akteuren, die über sozial geteilte Wissenskonstruktionen verfügen“ (S. 56) und Träger spezifischer Wissenskonstruk- 
tionen können sich ,nah“ oder „fern“ sein. Dieses Konzept wendet Heimann einerseits an, um bezogen auf den Klimawandel Gruppen mit ähnlichen Wahrnehmungsweisen zu finden, andererseits sucht er damit nach Erklärungen bzw. Faktoren für Unterschiede zwischen klimakulturellen Orientierungen, wie Werte, Weltbilder, Identitätskonstruktionen oder nationale Herkunft. Bei letzterer Frage bezieht er sich auf den raumtheoretischen Diskurs um kulturelle Globalisierung, in dem zum einen - insbesondere auch bezogen auf die globale Thematik des Klimawandels - ein Trend zu kultureller Homogenisierung und zum Entstehen einer Weltgesellschaft gesehen wird, zum anderen jedoch Positionen existieren, die auf die weiterhin große Relevanz lokaler Denk- und Handelsweisen verweisen.

In den Kapiteln 3 bis 6 setzt sich Heimann mit den zentralen Wissenskonstruktionen zu seinem Forschungsgegenstand auseinander. Dazu beschreibt er zunächst die Diskurse zu Problemen und Chancen durch den Klimawandel (Vulnerabilitätskonstruktionen, Kapitel 3) sowie zu Maßnahmen zu Klimaschutz und Klimaanpassung (Kernstrategien der Raumentwicklung bzw. Resilienzkonstruktionen, Kapitel 4). Es folgt eine literaturbasierte Heuristik zu Hypothesen für kulturelle Unterschiede bezüglich der Wahrnehmung des Klimawandels (Kapitel 5). Die Fallbeispiele für die Empirie stellen Küstenräume in Deutschland, Dänemark, Polen sowie den Niederlanden. Für diese Länder sowie global und für Europa betrachtet geht Heimann dann auf die politisch-administrativen Rahmenbedingungen ein (Kapitel 6), um so die jeweiligen spezifischen Kontexte als mögliche Faktoren zu illustrieren.

Für seine empirische Erhebung, in welcher der relationale Kulturraumansatz angewendet wird, führte Heimann eine Primärerhebung mittels Online-Fragebogen unter 830 Akteuren durch, die aus dem Bereich der Raumentwicklung - aus den Funktionsbereichen Verwaltung, Politik, Zivilgesellschaft und Wirtschaft - der jeweiligen vier untersuchten Küstenregionen kamen (Kapitel 7). Der Aufwand von Heimanns Erhebung ist bemerkenswert.

In den drei folgenden Kapiteln werden die Ergebnisse dargestellt. Für die Kommunikation über Klimawandel in den Untersuchungsregionen (Kapitel 8) - als eine Voraussetzung dafür, dass Wissen global geteilt werden kann stellt Heimann fest, dass von einer Transnationalisierung, nicht jedoch von einer Deterritorialisierung der Kommunikation über Klimawandel gesprochen werden kann. Hier lässt sich anmerken, dass sich zeitdiagnostische Thesen wie die, dass Kommunikation zunehmend global verläuft, mit einmaligen Querschnittsbefragungen nur begrenzt prüfen lassen.

Basierend auf seiner Empirie beschreibt Heimann dann zunächst unterschiedliche Klimakulturen an den europäischen Küsten hinsichtlich ihrer jeweils geteilten Wahrnehmung von Chancen und Risiken (Kapitel 9) und von Maß- nahmenpräferenzen (Kapitel 10) bezogen auf den Klimawandel. Es zeigt sich, dass es bei den Befragten keine einheitliche Problemkonstruktion bezüglich des Klimawandels gibt (auch wenn es ein Übergewicht bei den vom Klimawandel Überzeugten gibt). Heimann erstellt hier unterschiedliche Typen von Vulnerabilitätskonstruktionen. Gleiches tut er für die Konstruktionen von Resilienz, die durch unterschiedliche Präferenzen beim Umgang mit Klimawandel gekennzeichnet sind. Bei den Anpassungskulturen zeigen sich große Unterschiede bei den Präferenzen von Strategien je nach Art der Herausforderung (Fluten durch Meer oder Binnenwasser, Maßnahmen gegen Hitze und Sturm). Die jeweils erstellten Typologien stellen ein wertvolles Ergebnis der Dissertation dar, die sich in zukünftigen Forschungsarbeiten weiter verwenden und überprüfen lassen.

Für die Frage, welche Erklärungen es für die Unterschiede zwischen Klimakulturen gibt, kommt Heimann zu dem Ergebnis (ebenfalls in den Kapiteln 9 und 10), dass verschiedene Formen von Hintergrundwissen hier eine besonders zentrale Rolle spielen: Dies sind zum Beispiel ökologische Weltbilder (basierend auf Dunlap/ Catton 1979), die sich etwa auf die Präferenz technischer oder ökologischer Maßnahmen zum Schutz gegen Hochwasser auswirken. Andere Erklärungen liefern universalistisch-altruistische Orientierungen (für ökologieorientiertere Maßnahmenpräferenzen), Eigennutzorientierungen (Präferenzen für technische Lösungen) oder Traditions-/ Konformitätsorientierungen (erhöhen die Wahrscheinlichkeit klimaskeptischer Positionen). Sicherheitsorientierungen liefern überraschenderweise kaum Erklärungen für Unterschiede. Dieses integrierte Erklärungsmodell stellt eine besondere Leistung der Arbeit dar, da es sich von Studien abhebt, die nur auf einzelne Faktoren fokussieren.

Heimanns Ergebnis zu der im raumtheoretische Diskurs um kulturelle Globalisierung diskutierten Frage, ob der $\mathrm{Zu}$ sammenhang zwischen Ort und Region auf der einen und Kultur auf der anderen Seite an Bedeutung verloren habe, lautet, dass differenziert werden muss: Je nach untersuchtem Aspekt können mal regionale Kontextfaktoren ausschlaggebend für Unterschiede sein, mal Erklärungen nach dem relationalen Raumverständnis. So zeigten sich bei den Problemkonstruktionen des Klimawandels nationale Unterschiede zwischen Polen auf der einen und den drei anderen Ländern auf der anderen Seite. Beim Thema Küstenschutz waren dagegen die Ähnlichkeiten zwischen Deutschland und Polen größer als im Vergleich zu Dänemark und den Niederlanden. Bei der Feldzugehörigkeit wiederum zeigten sich vor allem Unterschiede bei den Wahrnehmungsweisen der Akteure aus den Nichtregierungsorganisationen gegenüber denen der Akteure aus Verwaltung, Politik und Wirtschaft. Es zeigt sich, dass es bei Kulturvergleichen wichtig ist, die Eigenart eines jeden untersuchten Problems zu berücksichtigen. 
Insgesamt leistet Thorsten Heimann mit seiner Dissertation einen wichtigen Beitrag zu den umweltsoziologischen, kultur- bzw. wissenssoziologischen sowie raumtheoretischen Debatten. Herauszuheben ist unter anderem - als ein übertragbar anwendbares Ergebnis - die Entwicklung eines integrierten und multiplen Erklärungsmodells für Unterschiede bezüglich der Wahrnehmung des Klimawandels und von Maßnahmen zur Anpassung an den Klimawandel. Die unvoreingenommene Differenziertheit der Ergebnisse - die nur durch eine größere Breite der berücksichtigten Faktoren und damit einen hohen Aufwand möglich wurde - ist eine der Kernleistungen der Arbeit.

\section{Literatur}

Berger, P. L.; Luckmann, T. (1966): Die gesellschaftliche Konstruktion der Wirklichkeit. Eine Theorie der Wissenssoziologie. Frankfurt am Main.

Brand, K.-W. (1995): Der ökologische Diskurs. Wer bestimmt Themen, Formen und Entwicklung der öffentlichen Umweltdebatte? In: de Haan, G. (Hrsg.): Umweltbewußtsein und Massenmedien. Perspektiven ökologischer Kommunikation. Berlin, 47-62.

Dunlap, R. E.; Catton, W. R. (1979): Environmental Sociology. In: Annual Review of Sociology 5, 243-273.

Knoblauch, H. (2010): Wissenssoziologie. Konstanz.

Reckwitz, A. (2000): Die Transformation der Kulturtheorien. Zur Entwicklung eines Theorieprogramms. Weilerswist.

Reusswig, F. (2010): Klimawandel und Gesellschaft. Vom Katastrophen- zum Gestaltungsdiskurs im Horizont der postkarbonen Gesellschaft. In: Voss, M. (Hrsg.): Der Klimawandel. Sozialwissenschaftliche Perspektiven. Wiesbaden, 75-97. 